Unfallchirurg $2021 \cdot 124: 5-6$

https://doi.org/10.1007/s00113-020-00887-3

(C) Springer Medizin Verlag GmbH, ein Teil von Springer Nature 2020

\section{Constanze Hausteiner-Wiehle ${ }^{1,2} \cdot$ Ludwig Lins $^{3}$}

${ }^{1}$ Psychosomatik, Neurozentrum, BG Unfallklinik Murnau, Murnau, Deutschland

${ }^{2}$ Klinik und Poliklinik für Psychosomatische Medizin und Psychotherapie, Klinikum rechts der Isar der Technischen Universität München, München, Deutschland

${ }^{3}$ Psychotraumatologie und Neuropsychologie, BG Unfallklinik Murnau, Murnau, Deutschland

\title{
Verletzt werden, verletzt bleiben: psychische Unfallfolgen
}

Menschen erleiden Sport-, Verkehrs-, Arbeits- oder Haushaltsunfälle, aber auch Überfälle, Folter, Krieg, Flucht oder Naturkatastrophen. Neben körperlichen können dabei psychische Strukturen und Funktionen verletzt werden, also das Erleben, Denken und Handeln. Dimensionen wie Affektregulation, Schmerzen und Gedächtnis zeigen, dass „Körperliches" und "Psychisches" dabei gar nicht eindeutig voneinander getrennt werden können.

Chirurgen sind oft die ersten, keineswegs nur die erstbesten, sondern sogar - zumindest anfangs - die besten Gesprächspartner für Verunfallte: Sie kennen Verletzungsmuster und Prognose, strahlen Zuversicht und Machbarkeit aus. Sie können merken, wenn „etwas nicht stimmt“. Das aktuelle Schwerpunktthema stellt deshalb psychische Unfallfolgen speziell für Unfallchirurgen dar: Jörg Angenendt öffnet den Blick dafür, wie vielfältig sie sind, wer gefährdet, wer geschützt ist, und welche Versorgungsangebote es gibt. Utz Ullmann stellt die Kernkategorie der letztlich im Vollbild gar nicht so häufigen, im Einzelfall aber besonders schwer verlaufenden posttraumatischen Belastungsstörung dar, ihre Phänomenologie, ihre Risikofaktoren und Strategien von Früherkennung und Basismanagement. Weil Psychotherapie oft wie ein Buch mit sieben Siegeln wirkt, geht Martin Sack näher auf konfrontative Traumatechniken ein. Ludwig Linsl sensibilisiert für direkte und indirekte, oft subtile und unterschätze kognitive und psychoemotionale Folgen von Schädel-Hirn-Traumata. Und weil Unfälle zu bleibenden psychischen Schäden führen können, die Frage nach dem Unfallzusammenhang aber oft strittig ist, erklärt Gordon Krahl gutachterliche Aspekte. Als Resümee lässt sich festhalten:

- Es lohnt sich, den Unfallhergang zu verstehen, um dessen psychischen „impact“ abschätzen zu können. Neben direkten Psychotraumata ergeben sich indirekte psychische Belastungen aus körperlichen Akutverletzungen und deren Langzeitfolgen. Ein glimpflich ausgegangener Forstunfall unter guten Kollegen wirkt geringer auf die Psyche ein als die Vermutung einer hohen Querschnittlähmung, ein bewaffneter Raubüberfall mit Mordandrohung, ein Verkehrsunfall, bei dem das Gehirn starken Scherkräften ausgesetzt ist oder bei dem das eigene Kind stirbt.

- Es lohnt sich, zumindest in groben Zügen den Kontext des Verletzten und den aktuellen psychopathologischen Befund zu kennen, um diejenigen Patienten zu identifizieren, die einer spezifischen (Mit-)Behandlung bedürfen. Vulnerabilität, Vorgeschichte und bisheriger Krankheitsverlauf beeinflussen Art, Ausmaß und Prognose psychischer Unfallfolgen beispielsweise Persönlichkeitszüge, Erfahrungen, Motive oder aktuelle Konflikte. Auch die Zeit, die seit dem Unfall verstrichen ist, wirkt als Kontextfaktor, denn wie körperliche Verletzungsfolgen können auch psychische spontan ausheilen oder aber chronifizieren. Eine Beobachtung des Verunfallten kann z. B. Dissoziation, Verhaltensauffälligkeiten, aber auch 


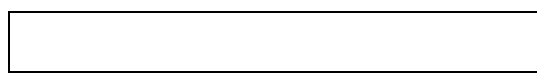

aggravationstypische Diskrepanzen aufzeigen.

- Es lohnt sich, Patienten mit einem gewissen (neuro-)psychologisch/ psychotherapeutischen Grundwissen gegenüber zu treten. Frühzeitige Interventionen wie empathisches Zuhören und Erklären, Stärkung von Motivation und Kraftquellen oder das Behandeln von Komorbiditäten sind oft einfach, effektiv und kostengünstig, verbessern Therapieadhärenz und Prognose. Gut nachvollziehbare Leib-Seele-Metaphern (ein Schreck „steckt noch in den Gliedern“ bzw. „im Nacken“, „raubt den Schlaf“, „hat den Boden unter den Füßen weggerissen“ oder „etwas in der Seele zerbrochen“) helfen, ins Gespräch zu kommen und weitere Optionen zu klären, z. B. eine psychologische Mitbetreuung. Sie helfen auch dabei, über einen Unfall hinwegzukommen, denn: „Zeit heilt Wunden“, „auch eine lange Reise beginnt mit dem ersten Schritt“, Menschen haben „ein dickes Fell“, können „Schrecken verdauen“ und „Herausforderungen schultern“.

In diesem Sinne,

Ihre

Constanze Hausteiner-Wiehle und Ludwig Linsl

\section{Korrespondenzadresse}

Prof. Dr. med. Constanze Hausteiner-Wiehle Psychosomatik, Neurozentrum, BG Unfallklinik Murnau

Prof.-Küntscher-Str. 8, 82418 Murnau,

Deutschland

c.hausteiner-wiehle@tum.de

Interessenkonflikt. C. Hausteiner-Wiehle und L. Lins geben an, dass kein Interessenkonflikt besteht.

\section{Lesetipp}

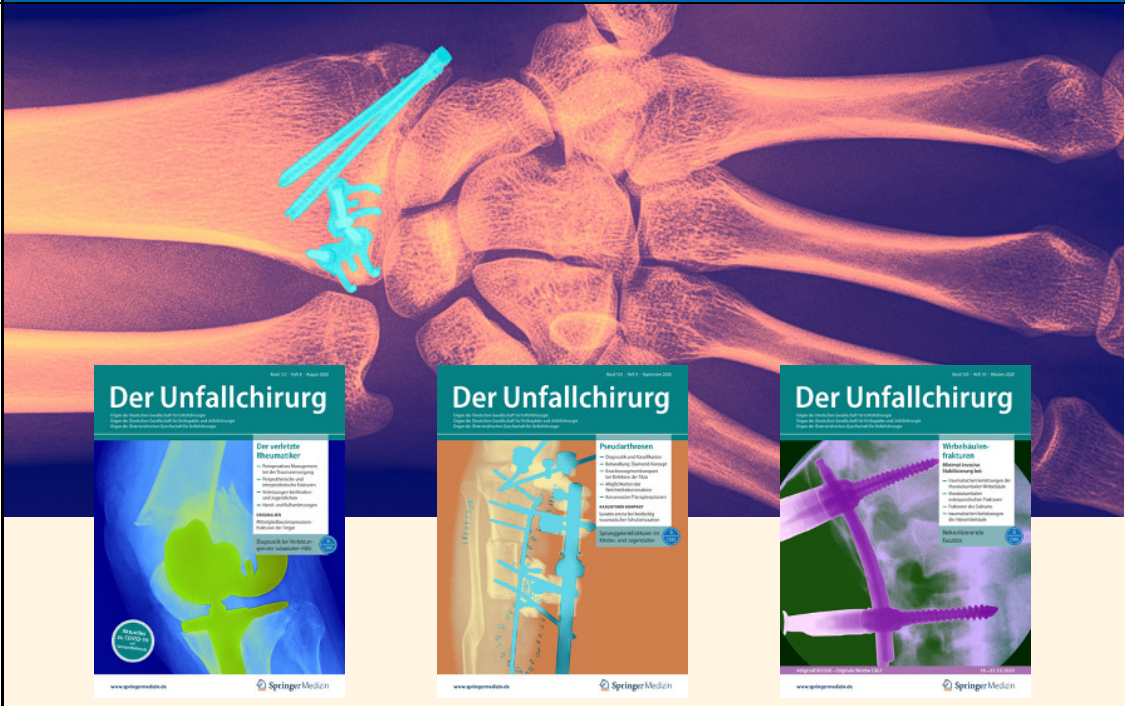

\section{Leitthemenübersicht von Der Unfallchirurg}

Der Unfallchirurg bietet Ihnen jeden Monat umfassende und aktuelle Beiträge zu interessanten Themenschwerpunkten aus allen Bereichen der Traumatologie.

Rückblick 2020

01/20 Klinisches Schaden- und Risikomanagement

02/20 Beugesehnenverletzungen

03/20 Blood Flow Restriction Training

04/20 Wirbelsäulenverletzungen im Kindesalter

05/20 Patient Reported Outcome Measurements (PROMs)

06/20 Interne Gefahrenlagen im Krankenhaus

07/20 Problemorientiertes Management 08/20 Der verletzte Rheumatiker

09/20 Pseudarthrosen

10/20 Wirbelsäulenfrakturen

11/20 Digitalisierung in O\&U

12/20 Die (schwer) verletzte schwangere Patientin

Alle Inhalte der Zeitschrift finden Sie unter www.springermedizin.de/

der-unfallchirurg

SpringerMedizin.de bietet Ihnen Zugang zu allen elektronisch verfügbaren Ausgaben Ihrer Zeitschrift - unabhängig davon, seit wann Sie die Zeitschrift abonniert haben.

\section{Vorschau 2021}

01/21 Psychische Unfallfolgen

02/21 Sehnenverletzungen an der Schulter

03/21 Frakturen des oberen Sprunggelenks

04/21 Strecksehnenverletzungen

05/21 Gelenkempyeme

06/21 Manuelle Medizin, Osteopathie und Ergotherapie

07/21 Sehnenverletzungen an Hüfte und Oberschenkel

08/21 Interventionelle Radiologie

09/21 Tumorbedingte pathologische Frakturen

10/21 Implantatversagen

11/21 Begutachtung im Kindesalter

12/21 Medizinische Drohnen

(Änderungen vorbehalten)

Unser Tipp: Mit dem e.Med-Abo können Sie jederzeit auf alle Inhalte von SpringerMedizin.de zugreifen. Auf Wunsch erhalten sie darüber hinaus eine gedruckte Fachzeitschrift ihrer Wahl.

Testen Sie e.Med 14 Tage lang kostenlos und unverbindlich!

\section{Jetzt informieren unter www.springermedizin.de/eMed}

